\title{
ALARM ROOT CAUSE DETECTION SYSTEM
}

\author{
Milan Rollo, Petr Novák, Jiř̌ Kubalík, Michal Pěchouček \\ Gerstner Laboratory, \\ Department of Cybernetics, Faculty of Electrical Engineering, \\ Czech Technical University in Prague, \\ Technická 2, 166 27, Prague 6, CZECH REPUBLIC \\ \{rollo|novakpe|kubalik|pechouc\}@labe.felk.cvut.cz
}

\begin{abstract}
Production process control becomes complicated as the complexity of the controlled process grows. To simplify the operator's role many computer based control systems with integrated visualization clients have been developed. In many practical circumstances malfunction of one or more process components results in other related components entering the alarm states. Several alarms appear on the operator's display in a short time making it difficult for the operator to diagnose the root cause quickly. Within this paper we describe a solution of this problem based on a multi-agent system that processes all incoming alarms, identifies the root cause alarms vs. alarms arising in consequence of the roots and presents the diagnostic results to the operator visually.
\end{abstract}

\section{INTRODUCTION}

Nowadays, as the complexity of the controlled processes grows, more stress is laid down on the operator. Number of manufacturers offers software for automatic process control with built-in visualization clients or even complex solutions including controllers, sensors, communication buses and visualization devices (e.g. control panels, touch screens). All these components a re dedicated to s upport the operator's role. They allow the operator to select the controlled component and assign limit values of variables to it. When the variable exceeds this value (or comes down under it) the operator is informed about it visually. This helps him to quickly recognize the origin of the problems and fix it up.

But the situation becomes more complicated for the operator when malfunction of single process component results in other related components entering the alarm states. In such a case several alarms may appear on operator's display simultaneously or in a short time and in a random order (which depends on the nature of the variables and speed of the sensors). Some of the alarms may not appear on the screen at all, because the control process is displayed schematically only and contains just principal components (due to the clarity reasons). This all makes it 
difficult for the o perator to recognize the origin of the problem. In this paper we describe a diagnostic system that supports the operator's decision making when such situation occurs. Main reason to develop this system was, that in some kinds of production processes is necessary to fix up the problems as soon as possible to avoid economical losses or eventual exposure to danger.

Solution is based on the multi-agent system that models the production process, processes all incoming alarms and determines the root cause. New determination algorithms, based either on the topology of the production process or physical nature of individual components, were developed to this purpose. Results of the determination process may be presented visually to the several operators. This diagnostic system nicely illustrates capabilities of the multi-agent system based solutions like a modularity or adaptability.

\subsection{State of the Art}

Use of multi-agent systems for modeling and controlling the production processes grows rapidly and seems to be very promising. In general the agents technologies are suitable for domains that posses either of the following properties: (i) where highly complex problems need to be solved or highly complex systems to be controlled or (ii) solving problems or controlling systems, where the information is distributed and is not available centrally. In manufacturing agent technologies have been applied mainly in planning highly complex production, control of dynamic, unpredictable and unstable processes, diagnostics, repair, reconfiguration and replanning. Important application domains of agent-based applications can be also found in the field of virtual enterprises (e.g. forming business alliances, forming long-term/short-term deals, managing supply chains) and logistics (e.g. transportation and material handling, optimal planning and scheduling, especially in cargo transportation, public transport but also peace-keeping missions, military maneuvers, etc.)

There are several companies that have adopted the agent-based solutions in production already, e.g. Daimler-Chrysler car manufacturing (McFarlane, 2000), Rockwell Automation developed agent based solution for BHP Melbourne, Australia, or ExPlanTech/ExtraPLANT agent-based production system running in Modelarna Liaz pattern factory, CZ and Hatzapoulos, packaging company, Greece (Pěchouček, 2002). The most relevant is the application of agent technology in a Reconfigurable Shipboard automation system developed by Rockwell automation and Rockwell Scientific Company (Maturana, 2003). In this application the agents are brought down to the level of physical components of the shipboard chilling system and they monitor and control stability of the whole of the chilling machinery in distributed manner. The practical applications are in the focus of attention of the HMS consortium (Brennan et al., 2003)

\section{SYSTEM DESCRIPTION}

Infrastructure of the proposed diagnostic system is shown schematically in Figure 1. Within this infrastructure, a software agent models each process component. Relationships of a gents represent the topology of the process components (theirs 
input/output links). Each agent receives alarms activated by the component it represents and collaborates with related agents to identify the potential root causes.

In order to do so effectively new detection algorithms, which utilize the process topology, component type and alarm type were developed. These algorithms complement the expert system (provided by the control software) and bring us a couple of advantages. Expert system based solutions require for each process control case separate knowledge base describing the relations and dependencies among the components. Such a knowledge base may not be available for the particular process or may contain incomplete information. Once an expert system is build up for a concrete process it provides an operator with most reliable results, however it doesn't cover the cases that occurred in the process never before. In contrast, the proposed agent based solution is general enough and is expected to work for an arbitrary manufacturing case.

The agent-based algorithms developed to date include rule-based and topologybased search methods, which appear to have promise for this purpose (see section 4).

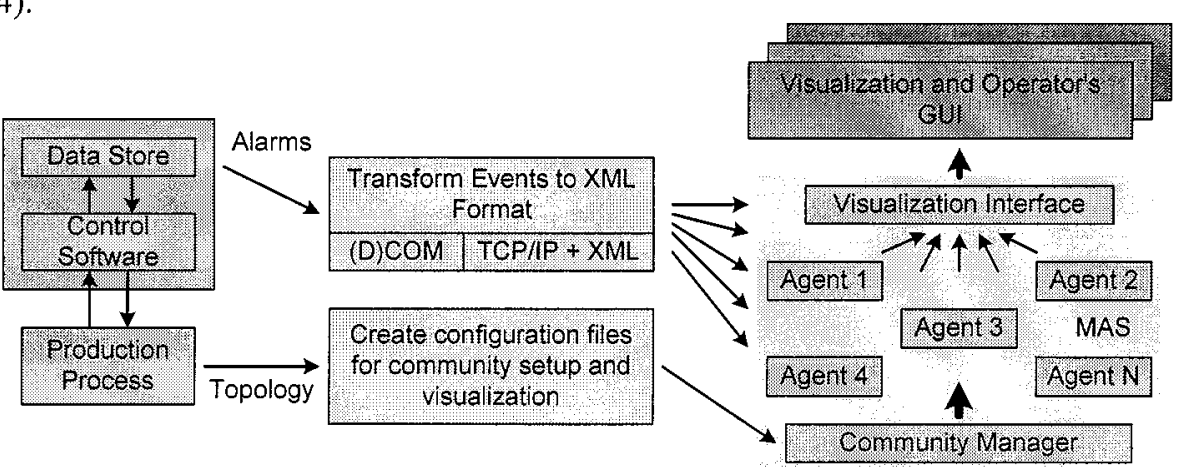

Figure 1 - Configuration of the multi-agent alarm root cause diagnosis system

\subsection{Production Process Interface}

In the past particular production processes were isolated and controlled by the software that used different forms of communication and data management. Technical progress in the area, especially the distributed control, brought couple of problems with interconnection among different production processes and control software. Industry leading companies thus formed a foundation dedicated to develop standard specifications. Most widely used solution and de facto standard in this area is currently the OPC (OLE for Process Control) (OPC, 2004).

OPC is based on the OLE/COM standard designed by Microsoft. For process automation systems that generate alarms and events the OPC Alarms and Events specification was developed. The OPC Alarms and Events server captures the alarms and events rose in the system, and makes them available to any client application that is interested in this information. It does not create the alarms and events; it only reports the alarms and events previously defined in the system using a standard communication interface (alarms and events in the system are automatically generated, based on the operating conditions and actions performed in the process plant). 
OPC in this case serves as a kind of database (Data Store) that all clients can utilize. Information is in this database filled by the control software that gets it from the sensors (e.g. through the communication bus). This database is shared among all clients.

In this project we use the OPC Alarms and Events server to receive the alarms invoked in the process (either real process or its simulation). These alarms (each containing information about its source, type, date of rise, variable, etc) are sent to the multi-agent system to be diagnosed.

\subsection{Multi-Agent System}

Alarm root cause determination process itself is carried out by the multi-agent system. Input of the determination process is an alarm record from the OPC server (converted to the XML format).

Multi-agent system brings us a couple of advantages compared with using an expert system only:

- Prediction ability - system can predict the problems (determine root causes) in process only from the general rules or process topology and thus discover problems that appeared never before and will not be covered by the expert system rules.

- Adaptability - system can be easily fit on different process (unlike expert system that is tied to the concrete process).

- Modularity - process can be simply extended with a new type of the component. Adding some new rules based on the physical characteristics of this component we can predict the behavior of the process (even when we have no knowledge about the entire process). Using the expert system, user is enforced to add a concrete rules based on the observation of the process's behavior.

\subsection{Operator's Visualization Interface}

Result of the root cause detection algorithm is represented by the change of agents' output state. This should be displayed on the operator's screen in a comprehensive way. When a complex system is to be controlled a number of output windows providing different views and details of the process may be required. For this purpose a versatile visualization system (VISIO) has been developed. It enables among others to define multiple snapshots of different parts of the process; they can be displayed to the operator either all at the same time or just some of them.

Developed visualization system contains also multiple-operator support. Each operator can be informed only about events that happened in the part of the production process he is responsible for.

\section{MULTI-AGENT SYSTEM IMPLEMENTATION}

Agent community consists of four different types of agents (see Figure 2). Beside the three static agents (Agent Factory, Root Cause Analysis Agent and Visualization 
Agent), each of them appears in the system only once, there are several Block Agents. Their number depends on the particular production process.

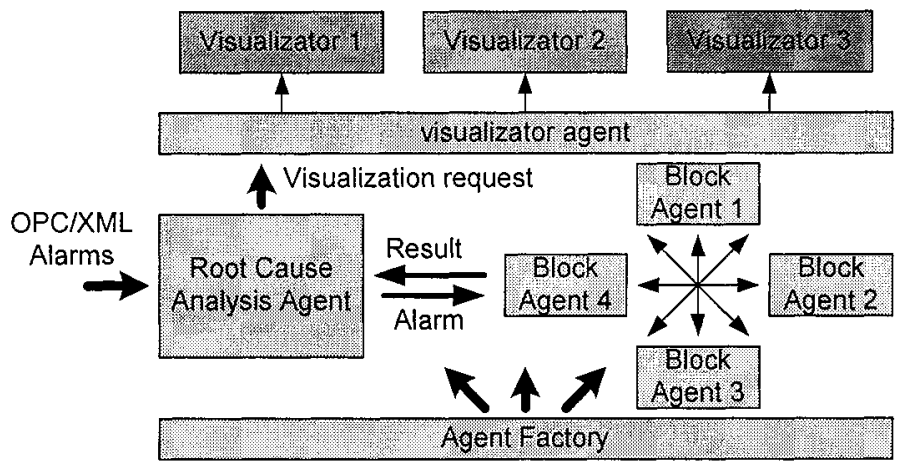

Figure 2 - Design of the multi-agent system

\subsection{Graph Representation}

Every component (pump, valve, tank, controller, etc.) is represented by one agent (Block Agent). Agents are connected by oriented links representing input/output relationships among components. Whole process thus forms an oriented graph. Detection algorithms utilize this feature during the communication among agents (prevention of circular communication inside the control loops).

Community manager (Agent Factory) generates agents according to the configuration file that contains record for each component: its name, agents linked to its input/output, information whether it generates alarms or not, type of component it represents (e.g. sensor, valve), the variable it monitors, etc. Note that the links connecting agents represent both material flow as well as control links present in the process (e.g. feed back control signals). This is important to make sure that no causal relation among components would be omitted when looking for the root cause alarm.

\subsection{Agent Types}

- Agent Factory - community manager used to startup the agent's community. It starts the agent platform (providing the necessary infrastructure), Root Cause Analysis Agent, Visualization Agent and certain number of Block Agents.

- Root Cause Analysis Agent (RCAA) - coordinator of the alarm determination process. It is connected to the OPC-XML interface and receives from the OPC Alarms and Events server all alarms that arose in the process. Alarms are then stored in the internal queue and processed on the FIFO basis. Each alarm is compared with the rules in the internal expert system first. In case that no matching rule is found, appropriate agent (corresponding to the source of alarm in the process) is informed about the event. Agents process the alarm using one of the inference modes and RCAA receives the result. Request to display this result on the operators' screens is then send to the Visualization Agent.

- Visualization Agent - is responsible for displaying the information about alarm states of all function blocks on the screens of all operators that are involved in. 
Agent connects to the VISIO program (see section 2.3) that is running on the operator's side. Once the information about change of function block's alarm state arrives from the RCAA, Visualization Agent transforms it into format acceptable by the VISIO and sends it to all operators that has appropriate function block on their screen.

- Block Agent - every Block Agent represents a real physical entity in the process. Block Agent has a record about its inputs, outputs and its alarm state. It has also assigned functionality in the process (e.g. pump, valve, tank, etc.). When agent receives information about new alarm it invokes an inference process to determine the alarm type.

\section{INFERENCE MODES}

Multi-agent system includes two inference modes: topology-based search and rulebased search. Topology-based search is based only on the topology of the process, rule-based exploits some background (prior) knowledge about the process. This knowledge is stored in form of rules in the rules database.

\subsection{Topology-Based Search}

Topology-based search takes advantage of converting the process model into its graph representation. This algorithm is based on the input/output relationship only and doesn't take into account features of the particular process. This algorithm operates in two separate phases:

\section{Backward (Upstream) Search:}

- Starting from the currently processed function block, it goes back through all its inputs (as long as the block has some or until the loop is closed).

- Looks for the possible cause of the alarms.

- Algorithm runs until the first block with alarm in the chain is found (this block will be marked as root).

\section{Forward (Downstream) Search:}

- Informs all successors (all agents in the output direction) that they are not root alarms any more.

- Updates alarm types for visualization.

Finally the results of the both search phases are merged together. In this result each agent (function block) has its own record that contains its name, type of the determined alarm type and information whether the agent can generate alarm. These records are stored in a tree form.

All alarms are stored in a buffer as they come and are processed sequentially one by the other. Final result of the algorithm does not depend on the order in which the alarms are processed. 


\subsection{Rule-Based Search}

The topology in itself represents only the lowest-level information about the monitored process. This makes the topology-based algorithm independent on the physical nature of the process. On the other hand, the topology itself cannot provide sufficient background for correct root cause detection in many cases. It is obvious that some rules, which take into account the knowledge about the process principles, may help to make the root cause detection more reliable.

A rule-based search algorithm utilizes the fact that individual agents model a specific component type (e.g. pump, valve, tank, heat exchanger). Each rule consists of two parts (condition and action) and is based upon the type of alarming component, the alarm type and the component topology. For example, in the case the agent receives a high-pressure alarm from a tank it searches for possible root cause on the input (e.g. malfunctioned pump) and output of the tank (e.g. closed valve).

Each agent has assigned a type of the component it represents (sensor, pump, valve, etc.), only agents that represent sensors can signal alarm. Each sensor has assigned a variable it monitors (pressure, temperature, etc.). When new alarm arises, rule-based search is invoked (provided that expert system fails to find a solution). This means that the corresponding agent searches its rule database for any rule whose condition part can be satisfied. If agent finds such a rule, it carries out actions specified in the action part of the rule. In case the agent cannot find any rule that could be applied the topology-based detection algorithm is initiated. Thus the topology-based search is considered to be a complementary option to the rule-based one. It is invoked only when the rule-based search fails to run.

\section{EXPERIMENTS}

The proposed diagnostic system was originally developed to improve the control process in hydrogen production plant. Because this process is very complex, simplified case study based on the distillation column was chosen to carry out the experiments (both processes have similar physical characteristics).

Instead of connection to the real physical process, mathematical model of the distillation column running in the Hysys (Hysys, 2004) simulation environment was used. This allowed us to better simulate the malfunctions and alarm explosions in the process. The DeltaV Automation System (DeltaV, 2004) was used as control software. Simulation environment and control software were connected via the OPC Server. Developed multi-agent diagnostic system was also connected to this server in order to receive the alarm records.

Distillation column used in this case study consists of more then fifty components (function blocks). We also carried out some additional scalability tests (measuring the number of messages and operational memory consumption), with several hundreds of components.

Multi-agent system was implemented in the Java using the JADE multi-agent platform (Jade, 2004). Other parts (Production Process Interface and Operator's Visualization Interface) were implemented in $\mathrm{C} / \mathrm{C}++$. 


\section{CONCLUSIONS AND FUTURE WORK}

System described in this paper illustrates the capabilities of the multi-agent systems to solve problems in highly complex distributed environment. Some of the important features of multi-agents systems we utilize in this case are modularity and adaptability. System can be easily reconfigured or extended with new elements according to the changes in the real production process.

Obviously, root cause alarm diagnostics based purely on the topology knowledge about the process $c$ annot r eliably r eturn the optimal s olutions b ecause it d oes not take into account the physical principle of the process. As the preliminary experiments showed the utilization of rule based search algorithm might considerably improve the performance of the diagnostic system.

There are still some open issues that need to be solved to make the diagnostic system more robust and reliable:

- Synchronization and cooperation between both inference modes (topologybased and rule-based search).

- Improve the connection with OPC Alarms \& Events server - suppression of alarms identified as a consequence of the root alarm in the DeltaV.

- Increase of alarm priorities (operator's notification level) if alarm isn't suppressed within a certain period of time (e.g. when high pressure alarm on the distillation column will arise, alarm priority will be increased each minute until the alarm will be suppressed).

\section{ACKNOWLEDGEMENT}

The project work has been in part co-funded by NASA Hydrogen Research Effort Software Agents and Knowledge Discovery and Data Mining Research for Complex System Safety, Health, and Process Monitoring project and by the Grant No. LN00B096 of the Ministry of Education, Youth and Sports of the Czech Republic.

\section{REFERENCES}

1. Brennan R., Hall K., Mařík V., Maturana F., and Norrie D. A real-time interface for holonic control devices. In Mařík, McFarlane, and Valckenaers, editors, Holonic and Multi-Agent Systems for Manufacturing, number 2744 in LNAI, pages 25-34. Springer-Verlag, Heidelberg, June 2003.

2. DeltaV - the Digital Automation System for Process Control. http:/www.easydeltav.com, 2004.

3. HYSYS - Integrated Simulation Environment. http://www.hyprotech.com/hysys, 2004.

4. JADE - Java Agent Development Framework. http://jade.tilab.com, 2004.

5. Maturana F., Tichý P., Šlechta P., and Staron R. A highly distributed intelligent multiagent architecture for industrial automation. In Mařik, Muller, and Pěchouček, editors, Multi-Agent Systems and Applications III, number 2691 in LNAI, pages 522-532. Springer-Verlag, Heidelberg, June 2003.

6. McFarlane D. and Bussmann S. Developments in holonic production planning and control. International Journal of Production Planning and Control, 11(6):552-536, 2000.

7. OPC Foundation. http://www.opcfoundation.org, 2004.

8. Pěchouček M., Řiha A., Vokřinek J., Mařík V., and Pražma V.. Explantech: applying multi-agent systems in production planning. International Journal of Production Research, 40(15):3681-3692, 2002 . 